Bull. Austral. Math. Soc.

20D30, 20E15

Vol. 64 (2001) [479-486]

\title{
SYLOW PERMUTABLE SUBNORMAL SUBGROUPS OF FINITE GROUPS II
}

\author{
A. BAllester-Bolinches and R. Esteban-Romero
}

In this paper a local version of Agrawal's theorem about the structure of finite groups in which Sylow permutability is transitive is given. The result is used to obtain new characterisations of this class of finite groups.

\section{INTRODUCTION}

Throughout the paper, the word group means finite group.

A subgroup $H$ of a group $G$ is said to be $S$-permutable in $G$ if $H$ permutes with every Sylow subgroup of $G$. By the results of Kegel [9] and Schmid [12], every S-permutable subgroup is subnormal and the set of all S-permutable subgroups is a sublattice of the lattice of all subnormal subgroups of $G$.

We say that a group $G$ is a $P S T$-group when the above lattices coincide, that is, when every subnormal subgroup is $S$-permutable. It follows that a group $G$ is a $P S T$-group exactly when the S-permutability is a transitive relation. Subclasses of $P S T$-groups are the class of $P T$-groups or groups in which permutability is transitive and the class of $T$-groups or groups in which normality is transitive.

Soluble PST-groups were first studied by Agrawal [1] in 1975, and recently by Alejandre, the first author and Pedraza-Aguilera [2], the authors [3], and Beidleman and Heineken [5]. Soluble $P T$-groups have been investigated by Zacher [13] in 1964, and more recently by Beidleman, Brewster and Robinson [4]. $T$-groups have been widely studied $[6,8,10,11]$.

The paper [3] provides a unified viewpoint for the classes of soluble $P S T, P T$ and $T$ groups in terms of their Sylow structure. The approach we have been following started in a paper of Bryce and Cossey [6], where a local version of some of the results on $T$-groups was established. This approach was also followed in the papers $[2,5]$.

One of our purposes in this paper is to give a local version of the classical theorems of Gaschütz, Zacher and Agrawal by using the results of [3]. We also provide new local

Received 12th April, 2001

Supported by Proyecto PB97-0674-C02-02 and Proyecto PB97-0604 from DGICYT, Ministerio de Educación y Ciencia

Copyright Clearance Centre, Inc. Serial-fee code: 0004-9727/01 \$A2.00+0.00. 
characterisations of the soluble PST-groups motivated by the results of the recent paper by Beidleman and Heineken [5].

Let us start with the classical characterisation of soluble PST-groups.

THEOREM 1. (Agrawal) A group $G$ is a soluble.PST-group if and only if $G$ has an Abelian normal Hall subgroup $N$ of odd order such that $G / N$ is nilpotent and the elements of $G$ induce power automorphisms in $N$.

In that result, if we add ' $G / N$ nilpotent modular group,' we obtain Zacher's characterisation of soluble $P T$-groups [13], and if we put ' $G / N$ Dedekind,' we get Gaschütz's characterisation of soluble $T$-groups [8].

The above results indicate that the three classes are closely related. However, there is a very important difference between them: the Sylow structure. In the case of $T$-groups, the Sylow subgroups are Dedekind, and modular in the case of $P T$-groups. There are no restrictions on Sylow subgroups in the $P S T$-case because every nilpotent group is a $P S T$-group.

For a prime $p$, Bryce and Cossey [6] introduced the class $T_{p}$ of all soluble groups $G$ for which every subnormal $p^{\prime}$-perfect subgroup of $G$ is normal. They prove:

THEOREM 2. A soluble group is a $T$-group if and only if it is a $T_{p}$-group for all primes $p$.

Motivated by Theorem 2, Alejandre, the first author and Pedraza-Aguilera introduced in [2] the class $P S T_{p}$ of all soluble groups $G$ in which every subnormal $p^{\prime}$-perfect subgroup in $G$ permutes with every Hall $p^{\prime}$-subgroup of $G$. They have proved:

THEOREM 3. A soluble group $G$ is a PST-group if and only if $G$ is a $P S T_{p}$-group for all primes $p$.

As we have noted in [3], Theorem 3 holds equally if the word 'soluble' is replaced by ' $p$-soluble' in the definition of the class of $P S T_{p}$-groups. Note that [2, Theorem 6] also holds in the $p$-soluble universe. Therefore we have:

THEOREM 4. A group $G$ is a $P S T_{p}$-group if and only if $G$ is p-supersoluble and all its chief $p$-factors are $G$-isomorphic regarded as $G$-modules by conjugation.

In the paper [5], Beidleman and Heineken define, for a prime $p$, the class $T_{p}^{\prime}$ (respectively, $T_{p}^{\prime \prime}$ ) of all soluble groups $G$ for which every $p^{\prime}$-perfect subnormal subgroup of $G$ is permutable (respectively, S-permutable) and prove:

THEOREM 5. Let $G$ be a soluble group. Then

1. $G$ is a $P T$-group if and only if it is a $T_{p}^{\prime}$-group for all primes $p$.

2. $G$ is a $P S T$-group if and only if it is a $T_{p}^{\prime \prime}$-group for all primes $p$.

Combining Theorem 4 and Theorem 5 of [3], we have:

TheOREM 6. A p-soluble group $G$ is a $P S T_{p}$-group if and only if $G$ is either $p$ nilpotent, or $G$ has an Abelian Sylow p-subgroup $P$ and every subgroup of $P$ is normal 
in $N_{G}(P)$.

Using Theorem 6, we prove in this paper a structure theorem for $P S T_{p}$-groups which can be considered as the local version of Agrawal's theorem. Before beginning the presentation of that theorem, the reader should be aware of the notation that is used.

Let $p$ be a prime and let $\mathfrak{E}_{p^{\prime}} \mathfrak{S}_{p}$ be the saturated formation of all $p$-nilpotent groups. For each group $G$, we denote $G(p)$ the $\mathfrak{E}_{p^{\prime}} \mathfrak{S}_{p}$-residual of $G$, that is, the smallest normal subgroup of $G$ such that $G / G(p)$ is $p$-nilpotent.

THEOREM A. A group $G$ is a $P S T_{p}$-group if and only if

1. either $G$ is $p$-nilpotent, or

2. $G(p) / O_{p^{\prime}}(G(p))$ is an Abelian normal Sylow p-subgroup of $G / O_{p^{\prime}}(G(p))$ such that the elements of $G / O_{p^{\prime}}(G(p))$ induce power automorphisms in $G(p) / O_{p^{\prime}}(G(p))$.

A local version of Gaschütz's theorem is obtained by adding in 1. in Theorem A the condition ' $G$ has Dedekind Sylow p-subgroups.' A local version of Zacher's theorem is obtained by adding in 1 . in Theorem A the condition ' $G$ has modular Sylow $p$-subgroups.'

The result is used in the paper to give a succinct proof of Agrawal's theorem.

Let $p$ be a prime. In any group $G$ there is a unique maximum normal $p$-nilpotently embedded subgroup, denoted here by $Z_{p}(G)$. Hence $Z_{p}(G)$ is $p$-nilpotent and there is a $G$ invariant series in $Z_{p}(G)$ such that the chief factors whose order is a power of $p$ are central, while $G / Z_{p}(G)$ has no central normal p-subgroups. $Z_{p}(G)$ is the $\mathfrak{E}_{p^{\prime}} \mathfrak{S}_{p}$-hypercentre of $G([7$, IV, 6.8$])$, and it contains $Z_{\infty}(G)$, the hypercentre of $G$. As a consequence of Theorem A we have:


subgroup of $O_{p^{\prime}, p}(G) / O_{p^{\prime}}(G(p))$ and

$$
O_{p^{\prime}, p}(G) / O_{p^{\prime}}(G(p))=G(p) / O_{p^{\prime}}(G(p)) \times Z_{p}\left(G / O_{p^{\prime}}(G(p))\right)
$$

We say that a subgroup $H$ of $G$ is p-hypercentrally embedded (respectively, hypercentrally embedded) if $H /$ Core $_{G}(H) \leqslant Z_{p}\left(G /\right.$ Core $\left._{G}(H)\right)$ (respectively, $H /$ Core $_{G}(H) \leqslant$ $Z_{\infty}\left(G /\right.$ Core $\left.\left._{G}(H)\right)\right)$. Beidleman and Heineken [5] proved that a soluble group $G$ is a $P S T$-group if and only if every subnormal subgroup permutes with every Carter subgroup of $G$ and the subnormal subgroups are hypercentrally embedded in $G$. We prove in the following that the permutability with the Carter subgroups can be removed.

THEOREM B. A p-soluble group $G$ is a $P S T_{p}$-group if and only if every $p^{\prime}$-perfect subnormal subgroup of $G$ is p-hypercentrally embedded in $G$.

Corollary 2. A soluble group $G$ is a PST-group if and only if every subnormal subgroup of $G$ is hypercentrally embedded in $G$. 


\section{ProOFs}

Proof of THEOREM A: Let $G$ be a $P S T_{p}$-group and assume that $G$ is not $p$ nilpotent. Then $G(p) \neq 1$ and $p$ divides $|G(p)|$. Applying Theorem $6, G$ has an Abelian Sylow $p$-subgroup $P$ such that every subgroup of $P$ is normal in $N_{G}(P)$.

Since $G / O_{p^{\prime}}(G(p))$ inherits the hypotheses of $G$, we can assume without loss of generality that $O_{p^{\prime}}(G(p))=1 . G(p)$ is contained in $G^{\prime}$, the commutator subgroup of $G$, which is $p$-nilpotent because $G$ is $p$-supersoluble. Hence $G(p)$ is $p$-nilpotent and so $G(p)$ is a $p$-group contained in $P$. In particular, $G(p)$ is Abelian.

Note that $[7, I V, 5.18]$ still holds in the $p$-soluble universe. Consequently $G(p)$ is complemented in $G$ by every $p$-nilpotent projector. Let $D$ be one of them and assume that $p$ divides $|D|$. Then there exists a $p$-chief factor $A / B$ of $G$ such that $G(p) \leqslant B<A$. This implies that $A / B$ is central in $G$. By Theorem 4, all $p$-chief factors of $G$ are $G$-isomorphic. In particular, every chief factor below $G(p)$ is central, a contradiction. Consequently $D$ is a Hall $p^{\prime}$-subgroup of $G$ and $G(p)$ is a normal Sylow $p$-subgroup of $G$. Hence every subgroup of $G(p)$ is normal in $N_{G}(G(p))=G$.

Conversely, suppose that $G$ is either $p$-nilpotent or $G(p) / O_{p^{\prime}}(G(p))$ is an Abelian normal Sylow $p$-subgroup of $G / O_{p^{\prime}}(G(p))$ such that the elements of $G / O_{p^{\prime}}(G(p))$ induce power automorphisms in $G(p) / O_{p^{\prime}}(G(p))$.

If $G$ is $p$-nilpotent, then $G$ is $p$-supersoluble and all $p$-chief factors are central. Hence $G$ is a $P S T_{p}$-group by Theorem 4 .

Assume now that $G(p) / O_{p^{\prime}}(G(p))$ is an Abelian Sylow $p$-subgroup of the group $G / O_{p^{\prime}}(G(p))$ and every subgroup of $G(p) / O_{p^{\prime}}(G(p))$ is normal in $G / O_{p^{\prime}}(G(p))$. Let $H$ be a subnormal $p^{\prime}$-perfect subgroup of $G$ and let $D$ be a Hall $p^{\prime}$-subgroup of $G$. Denote $T=H O_{p^{\prime}}(G)$. Then $T=(T \cap G(p))(T \cap D)$ and $T \cap G(p) / O_{p^{\prime}}(G(p))$ is normal in $G / O_{p^{\prime}}(G(p))$. Therefore $T \cap G(p)$ is normal in $G$. Now $H D=T D=(T \cap G(p)) D$, which is a subgroup of $G$. Consequently $H$ permutes with $D$ and $G$ is a $P S T_{p}$-group.

Proof of Corollary 1: It is clear that we may assume that $G(p) \neq 1$ and $O_{p^{\prime}}(G(p))=1$. By Theorem A, $G(p)$ is an Abelian normal Sylow $p$-subgroup of $G$ and so $G(p) \leqslant O_{p^{\prime}, p}(G)$. Let $D$ be a Hall $p^{\prime}$-subgroup of $G$. Then $G=G(p) D$. Therefore $O_{p^{\prime}, p}(G)=G(p)\left(D \cap O_{p^{\prime}, p}(G)\right)$. Since $O_{p^{\prime}, p}(G)$ is $p$-nilpotent and $D \cap O_{p^{\prime}, p}(G)$ is a Hall $p^{\prime}$-subgroup of $O_{p^{\prime}, p}(G)$, it follows that $D \cap O_{p^{\prime}, p}(G)$ is normal in $G$. Consequently, $D \cap O_{p^{\prime}, p}(G) \leqslant C_{G}(G(p))$, which coincides with $Z_{p}(G)$ by [7, IV,6.14]. On the other hand, since $G(p)$ is Abelian, then $D$ is actually a $p$-nilpotent projector of $G$ by $[7, I V, 5.18]$. Since, by $[7$, IV, 6.14$], Z_{p}(G) \leqslant D$, it follows that $D \cap O_{p^{\prime}, p}(G)=Z_{p}(G)$. The proof of the corollary is now complete.

Proof of Agrawal's Theorem (Theorem 1): Suppose that $G$ is a PST-group. Let $\pi$ be the set of all primes $p$ such that $G$ has a non-central chief $p$-factor. Then

$$
\left.T=G^{\mathfrak{N}}=\langle G(p)| p \text { is a prime }\right\rangle=\langle G(p) \mid p \in \pi\rangle
$$


because $G(p)=1$ if $p$ does not belong to $\pi$. We prove that $T$ is a Hall $\pi$-subgroup of $G$. Since $G$ is supersoluble, we have that $G^{\prime}$ is nilpotent. Hence $T$ is nilpotent. Assume that $q$ is a prime dividing the order of $T$ such that $q \notin \pi$. Let $T_{q^{\prime}}$ be a Hall $q^{\prime}$-subgroup of $T$. Then $T_{q^{\prime}}$ is a normal subgroup of $G$. Since $q \notin \pi$, we have that all chief $q$-factors of $G$ are central. Therefore $G / T_{q^{\prime}}$ is nilpotent. Moreover, $T_{q^{\prime}}$ is a normal subgroup of $G$, and $G / T_{q^{\prime}}$ is nilpotent. This implies that $T=T_{q^{\prime}}$, and $T$ is a $q^{\prime}$-group, a contradiction. Consequently $T$ is a $\pi$-subgroup of $G$. If $p$ belongs to $\pi$, then $G(p)$ contains an Abelian Sylow $p$-subgroup of $G$ by Theorem A. Hence $T$ is Abelian. Moreover, 2 cannot belong to $\pi$, because a $P S T_{2}$-group is always 2-nilpotent. We must show now that every subgroup of $T$ is normal in $G$. Since $T$ is nilpotent, we may assume without loss of generality that $T$ is a $p$-group for some prime $p$. Then $O_{p^{\prime}}(T)=O_{p^{\prime}}(G(p))$ and $G(p)$ is a Sylow $p$-subgroup of $G$. Hence $G(p)=T$ and every subgroup of $T$ is normal in $N_{G}(T)$ by Theorem A.

Conversely, suppose that $N=G^{\mathfrak{M}}$ is an Abelian normal Hall subgroup $N$ of odd order of $G$ such that every subgroup of $N$ is normal in $G$. Then it is clear that $G$ satisfies the conditions of Theorem A for all primes $p$. Therefore $G$ is a $P S T_{p}$-group for all primes $p$ and then $G$ is a PST-group.

The following lemma is needed to prove Theorem $\mathrm{B}$.

Lemma 1. If $R$ is a Hall $p^{\prime}$-subgroup of a group $G$, then $Z_{p}(G) \leqslant N_{G}(R)$.

Proof: Let $G$ be a counterexample of minimal order to this lemma. Let $R$ be a Hall $p^{\prime}$-subgroup of $G$ such that $Z_{p}(G) \& N_{G}(R)$. Let $T=Z_{p}(G) R$. If $T$ is a proper subgroup of $G$, then $Z_{p}(G) \leqslant Z_{p}(T)$, which is contained in $N_{T}(R)$ by the minimal choice of $G$, a contradiction. Therefore $T=G$ and $G=Z_{p}(G) R$. Since $R$ is a $p^{\prime}$-group, $R$ is $p$-nilpotent. Let $X$ be a maximal $p$-nilpotent subgroup of $G$ containing $R$. By [7, IV,6.14], it follows that $Z_{p}(G) \leqslant X$. Consequently $G=X$ and $G$ is $p$-nilpotent. This implies that $R$ is a normal subgroup of $G$, final contradiction.

Proof of Theorem B: Suppose that $G$ is a $P S T_{p}$-group. Then, by Theorem 6, either $G$ is $p$-nilpotent, or $G$ has an Abelian Sylow p-subgroup $P$ and every subgroup of $P$ is normalised by the normaliser $N_{G}(P)$.

In the first case, if $G$ is $p$-nilpotent, every $p^{\prime}$-perfect subnormal subgroup is $p$ hypercentrally embedded. On the other hand, assume that a Sylow $p$-subgroup $P$ is Abelian and every subgroup of $P$ is normal in $N_{G}(P)$. Note that the proof of [6, Theorem 2.3] works in the $p$-soluble universe. Hence in this case every $p^{\prime}$-perfect subnormal subgroup of $G$ is normal. Consequently $H_{G}=H$ and $H / H_{G} \leqslant Z_{p}\left(G / H_{G}\right)$.

Conversely, suppose that every $p^{\prime}$-perfect subnormal subgroup $H$ of $G$ is $p$-hypercentrally embedded. Let $H$ be a subnormal $p^{\prime}$-perfect subgroup of $G$, and let $R$ be a Hall $p^{\prime}$-subgroup of $G$. Since $H / H_{G} \leqslant Z_{p}\left(G / H_{G}\right)$ and $R H_{G} / H_{G}$ is a Hall $p^{\prime}$-subgroup of $G$, it follows by Lemma 1 that $H / H_{G}$ normalises $R H_{G} / H_{G}$. In particular, $H$ permutes with $R H_{G}$. This implies that $H$ permutes with $R$. Therefore $G$ is a $P S T_{p}$-group.

Proof of Corollary 2: Suppose that $G$ is a $P S T$-group of minimal order with a 
subnormal subgroup $H$ not hypercentrally embedded in $G$. Assume that $H$ is of minimal order. We can clearly assume that $H_{G}=1$. Hence $H$ is nilpotent by [12, Proposition A] and every Sylow subgroup of $H$ is S-permutable in $G$ by [12, Proposition B]. From the minimality of $H$, we can suppose that $H$ is a $p$-group for a prime $p$. Since $G$ is a $P S T_{p^{-}}$ group, $H$ is $p$-hypercentrally embedded in $G$ by Theorem B. Since $H$ is a subnormal $p$-subgroup, its normal closure $K=\left\langle H^{G}\right\rangle$ is a $p$-group. Let $A / B$ be a chief factor of $G$ such that $B<A \leqslant K$. Since $B / A$ is a $p$-group and $K \leqslant Z_{p}(G)$, it follows that $B / A$ is central in $G$. Thus $K$ is contained in $Z_{\infty}(G)$. In particular, $H$ is hypercentrally embedded in $G$.

Conversely, suppose that every subnormal subgroup of $G$ is hypercentrally embedded in $G$. Let $p$ be a prime number and let $H$ be a $p^{\prime}$-perfect subnormal subgroup. Since $H$ is hypercentrally embedded in $G$, it follows that $H$ is $p$-hypercentrally embedded in $G$. Hence $G$ is a $P S T_{p}$-group by Theorem B. Therefore $G$ is a $P S T$-group by Theorem 3 .

\section{Examples}

EXAMPLE 1. The result of Corollary 1 cannot be sharpened to $O_{p^{\prime}, p}(G)=G(p) Z_{p}(G)$ when $O_{p^{\prime}}(G(p)) \neq 1$. The symmetric group $\Sigma_{3}$ of degree 3 has an irreducible and faithful module over the field of 2 elements. Let $V=\left\langle v_{1}, v_{2}\right\rangle$ and $W=\left\langle w_{1}, w_{2}\right\rangle$ be two $\Sigma_{3^{-}}$ isomorphic copies of these modules. There is an action of the cyclic group $C_{2}=\langle z\rangle$ of order 2 on $V \times W$ such that $v_{j}^{z}=w_{j}, w_{j}^{z}=v_{j}$ for $j \in\{1,2\}$. Hence we can consider the semidirect product $G=[V \times W]\left(\Sigma_{3} \times C_{2}\right)$ with respect to this action. This group is clearly a $P S T_{3}$-group, because its Sylow 3-subgroup has order 3, but

$$
\begin{aligned}
Z_{3}(G) & =1, \\
O_{3^{\prime}, 3}(G) & =(V \times W)\left(A_{3} \times C_{2}\right)
\end{aligned}
$$

and

$$
G(3)=(V \times W) A_{3},
$$

whence

$$
O_{3^{\prime}, 3}(G) \neq G(3) Z_{3}(G)
$$

EXAMPLE 2. One might ask whether a $p$-soluble group $G$ is a $P S T_{p}$-group if and only if every $p^{\prime}$-perfect subnormal subgroup of $G$ is hypercentrally embedded. The answer is negative: The dihedral group $D_{8}$ of order 8 has an irreducible and faithful module of dimension 2 over the field of 3 elements. Let $G$ be the corresponding semidirect product. This group is a $P S T_{2}$-group, because it is 2-nilpotent. On the other hand, $G$ possesses 2 -perfect core-free subnormal subgroups of order 6 isomorphic to the symmetric group $\Sigma_{3}$ of degree 3 . These subgroups cannot be hypercentrally embedded, because they are not nilpotent.

This example also shows that a $P S T_{p}$-group does not have property $T_{p}^{\prime \prime}$ in general. 
EXAMPLE 3. There are non-PST $T_{p}$-groups such that every subnormal $p$-subgroup is hypercentrally embedded. For instance, let $G=\langle x\rangle \times \Sigma_{4}$, where $\langle x\rangle$ is a cyclic group of order 3 and $\Sigma_{4}$ is the symmetric group of degree 4. The subnormal 3-subgroups of $G$ are 1 and $\langle x\rangle$. Both are hypercentrally embedded in $G$, because they are normal. But $G$ is not a $P S T_{3}$-group, because the 3 -factor $\langle x\rangle$ is central but $A_{4} / V_{4}$ is not.

EXAMPLE 4. Even in the case that all subnormal p-subgroups of $G$ are hypercentrally embedded for all $p$ we cannot ensure that a soluble group is a $P S T$-group: The cyclic group $C_{3}$ of order 3 has an irreducible and faithful module $V_{7}$ over the field of 7 elements. The group $C_{3} \times \Sigma_{3}$ acts on $V_{7}$ with kernel $\Sigma_{3}$. Let $G$ be the semidirect product corresponding to this action. The unique subnormal subgroups of prime-power order of $G$ are $1, A_{3}$ and $V_{7}$. All these subgroups are normal and so hypercentrally embedded. But $G$ is not a $P S T$-group, because the action of $C_{2} \leqslant \Sigma_{3}$ on $C_{3}$ is trivial, while the action on $A_{3}$ is non-trivial.

\section{REFERENCES}

[1] R.K. Agrawal, 'Finite groups whose subnormal subgroups permute with all Sylow subgroups', Proc. Amer. Math. Soc. 47 (1975), 77-83.

[2] M.J. Alejandre, A. Ballester-Bolinches, and M.C. Pedraza-Aguilera, 'Finite soluble groups with permutable subnormal subgroups', J. Algebra 240 (2001), 705-722.

[3] A. Ballester-Bolinches and R. Esteban-Romero, 'Sylow permutable subnormal subgroups of finite groups', preprint.

[4] J.C. Beidleman, B. Brewster, and D.J.S. Robinson, 'Criteria for permutability to be transitive in finite groups', J. Algebra 222 (1999), 400-412.

[5] J.C. Beidleman and H. Heineken, 'Finite soluble groups whose subnormal subgroups permute with certain classes of subgroups', (preprint).

[6] R.A. Bryce and J. Cossey, 'The Wielandt subgroup of a finite soluble group', J. London Math. Soc. 40 (1989), 244-256.

[7] K. Doerk and T. Hawkes, Finite soluble groups, de Gruyter Expositions in Mathematics 4 (Walter de Gruyter, Berlin, New York, 1992).

[8] W. Gaschütz, 'Gruppen, in dennen das Normalteilersein transitiv ist.', J. Reine Angew. Math. 198 (1957), 87-92.

[9] O.H. Kegel, 'Sylow-Gruppen und Subnormalteiler endlicher Gruppen', Math. Z. 78 (1962), 205-221.

[10] J.C. Lennox and S.E. Stonehewer, Subnormal subgroups, Oxford Mathematical Monographs (Oxford University Press, New York, 1987).

[11] D.J.S. Robinson, 'A note on finite groups in which normality is transitive', Proc. Amer. Math. Soc. 19 (1968), 933-937.

[12] P. Schmid, 'Subgroups permutable with all Sylow subgroups', J. Algebra 207 (1998), 285-293.

[13] G. Zacher, 'I gruppi risolubli finiti in cui i sottogruppi di composizione coincidono con i sottogrupi quasi-normali', Atti Accad. Naz. Lincei Rend. Cl. Sci. Fis. Mat. Natur. 37 (1964), 150-154. 
Departament d'Àlgebra

Universitat de València

Dr. Moliner, 50

E-46100 Burjassot (València)

Spain

e-mail: Adolfo.Ballester@uv.es
Departament de Matemàtica Aplicada Universitat Politècnica de València

Camí de Vera, $\mathbf{s} / \mathbf{n}$

E-46022 València

Spain

e-mail: resteban@mat.upv.es 